\title{
Minimally invasive restorative treatment with direct composite veneers - case report
}

\author{
Sonja Apostolska', Vasilka Rendzova', Marina Eftimoska', Vesna Filipovska', Sasho Elenchevski², \\ Nadica Janeva ${ }^{2}$ \\ 'University "Ss. Cyril and Methodius", Faculty of Dental Medicine, Department of Restorative Dentistry and \\ Endodontics, Skopje, Republic of Macedonia; \\ 2University "Ss. Cyril and Methodius", Department of Mobile Prosthodontics, Faculty of Dental Medicine, Skopje, \\ Republic of Macedonia
}

\begin{abstract}
SUMMARY
Esthetic and minimally invasive restorative techniques are current topics in dentistry. Introduction of modern composite materials and adhesive technology provided numerous options for design of esthetic restorations without invasive teeth preparation common for traditional prostodontic restorations. Modern esthetic dentistry can provide solutions for simple as well as complex cases by implementation of various alternatives.

Keywords: veneers; adhesive technique; composite materials
\end{abstract}

\section{INTRODUCTION}

Esthetic and minimally invasive restorative techniques are current topics in dentistry. Introduction of modern composite materials and adhesive technology provided numerous options for design of esthetic restorations without invasive teeth preparation common for traditional prostodontic restorations. Modern esthetic dentistry can provide solutions for simple as well as complex cases by implementation of various alternatives $[1,2]$.

The need for esthetic treatment emerges from different systemic preconditions as well as various genetic, dental and traumatic reasons $[3,4,5]$. Key factors for treating these cases are composite materials and adhesive systems. Layered technique using different shades produces excellent bio-mimetic restorations. By utilizing positive characteristics of these materials high esthetic and functional effects can be achieved [6].

Modern composites allow reconstruction of the outer forms and colors but also reproduce inner tooth structure. Esthetic restoration mimics optical characteristics of natural tooth $[7,8]$. Nano-technology of composite materials has simpler color structure and very intense 'chameleon effect'. It allows excellent modeling of the filling and complex teeth restoration in anterior and posterior regions. Clinical procedure for direct composite restoration involves: respecting individual morphological characteristics of teeth, choosing appropriate shades, proper teeth preparation, anatomic layered technique and final finishing and polishing. Applying layered technique provides anatomical teeth form, however, requires long application time. On the other hand, layered technique sometimes traps air causing voids in the composite that later became stained. That lowers esthetical characteris- tics of restorations over time $[9,10]$. These problems can be overcome with new composite systems that allow easy restoration of anterior teeth by the application of alreadymade composite veneers. This is convenient when changing shape of the tooth is also required. Excellent results have been reported in restoring fractured and destroyed teeth, morphological and structural deviations, discolored teeth and diastema, caries, trauma, erosion, abrasion, fractures and minor orthodontic deviations $[11,12]$. Direct composite systems offer treatment that is both for the patient and dentist more economical and can be finished during only one visit.

The aim of this is to present two clinical cases of anterior teeth reconstruction using Componeer system.

\section{Case 1}

A female patient, 40 years old, visited the Clinic for Restorative Dentistry and Endodontics dissatisfied with the appearance of her teeth in the upper anterior region. During the clinical check up of her teeth 13, 12, 11,21,22, 23 inadequate morphology and restorations were found (Figure 1). Proposed treatment included restoring teeth

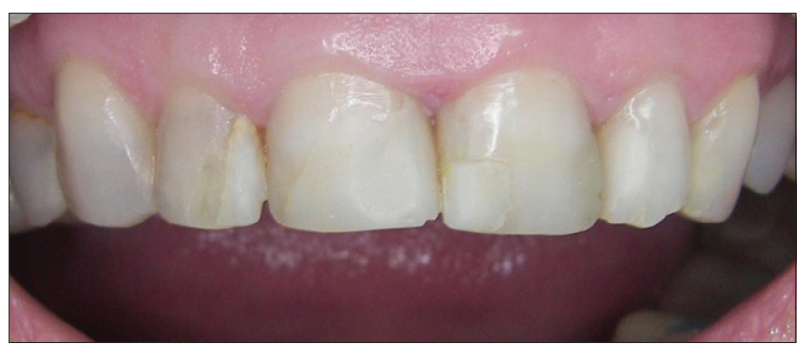

Figure 1. Teeth before the procedure

Slika 1. Izgled zuba pre početka terapije 


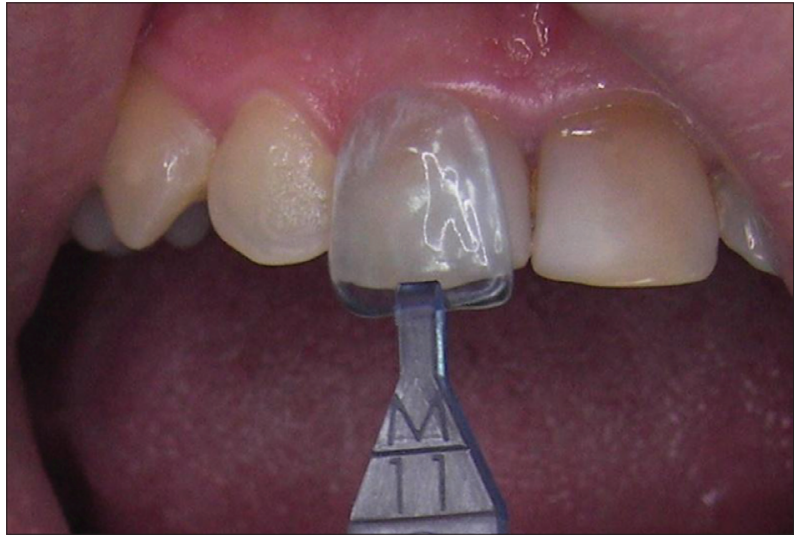

Figure 2. Form selected with ComponeerTM Contour Guide that is unique, transparent, precise contour in order to select proper tooth form

Slika 2. ComponeerTM Contour Guide za precizno određivanje kontura i oblika zuba

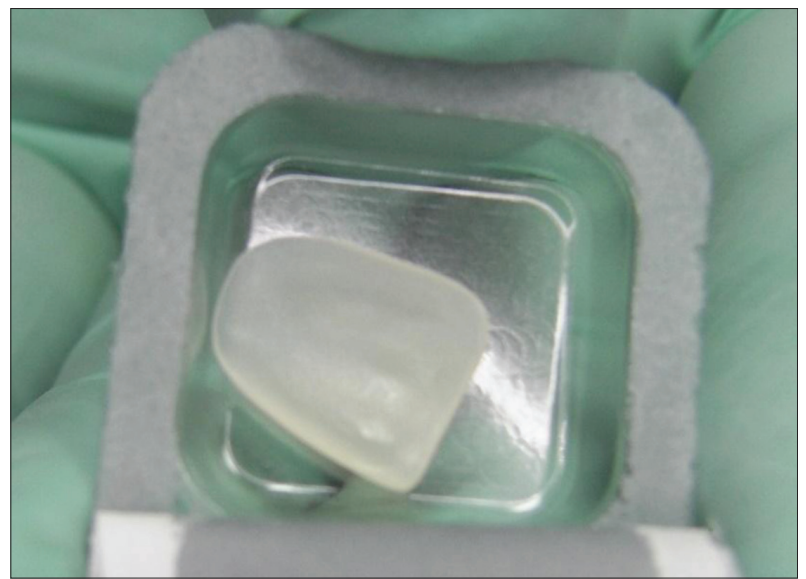

Figure 3. Composite veneer

Slika 3. Kompozitna faseta

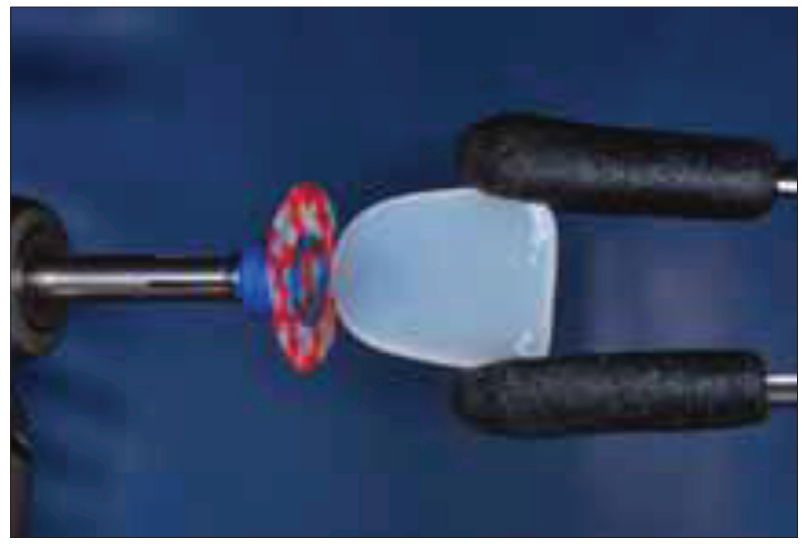

Figure 4. Veneer correction using round disk (Swiss Flex, Coltene) Slika 4. Korekcija fasete okruglim diskom (Swiss Flex, Coltene)

with the Componeer system. After cleaning the teeth surface, dentin and enamel colors were selected using the color key under the natural light. ComponeerTM Contour Guide is unique and transparent precise contour for the selection of the proper tooth form and size L was chosen in this case (Figures 2,3). Further on, the selected enamel veneer was modified using round disk (Swiss Flex, Coltene) with low rotation without any water due to the marginal adaptation (Figure 4). Afterwards,

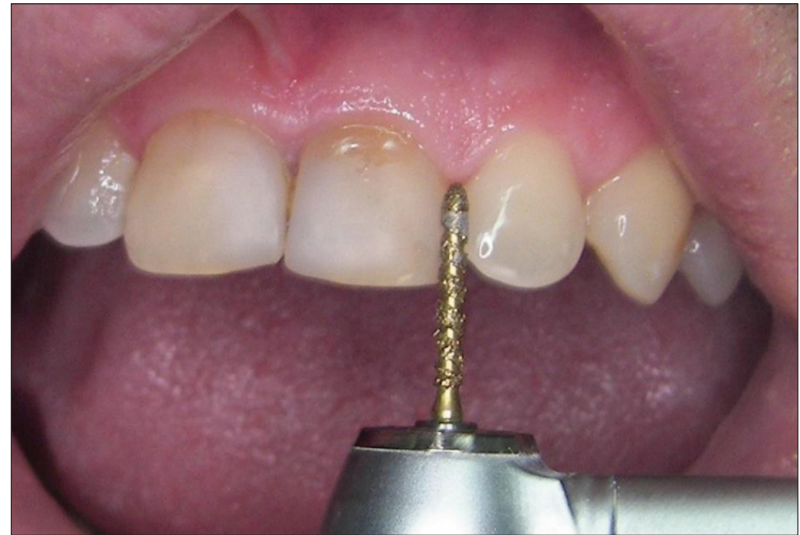

Figure 5. Minimal tooth preparation Slika 5. Minimalna preparacija zuba

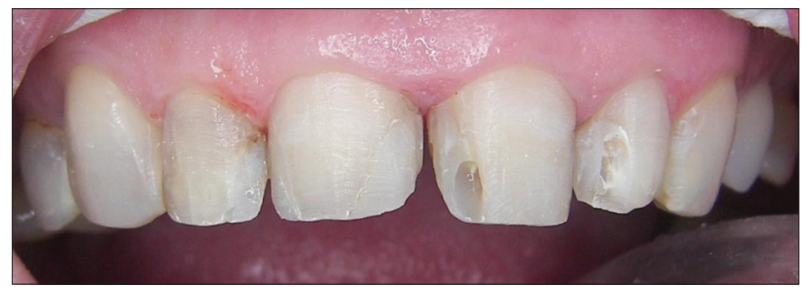

Figure 6. Removal of previous restorations Slika 6. Uklanjanje starih restoracija

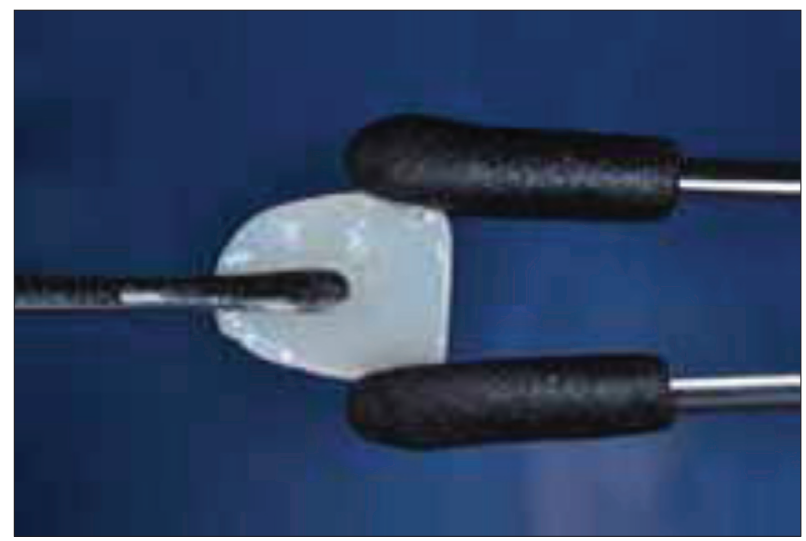

Figure 7. BRILIANT EverGlow A2/B2 enamel color Slika 7. Gleđna boja BRILIANT EverGlow A2/B2

minimal preparation was done on the teeth, as the veneer thickness was only $0.3 \mathrm{~mm}$. Old restorations were also removed (Figures 5 and 6). One Coat Bond was applied on the inner surface of the Componeer veneer and left without light polymerization. The teeth were etched with $35 \%$ phosphoric acid for 30 seconds and rinsed, and then One Coat Bond on the teeth surface was applied, dried and polymerized for 20 seconds. Composite color BRILLIANT EverGlow A2/B2 was applied on the teeth, whereas the enamel color was applied on the inner surface of Componeer veneer with a specialized spatula without any pressure. The composite veneer was hold with a specially designed holder. Then it was applied to the tooth by putting pressure on a specialized silicone instrument (Figures 7,8$)$. After checking the position, occlusion and the central line of incisors the palatal side was polymerized first (due to lowering polymerization stress) and then labial side. Polymerization was 40 seconds long both on 


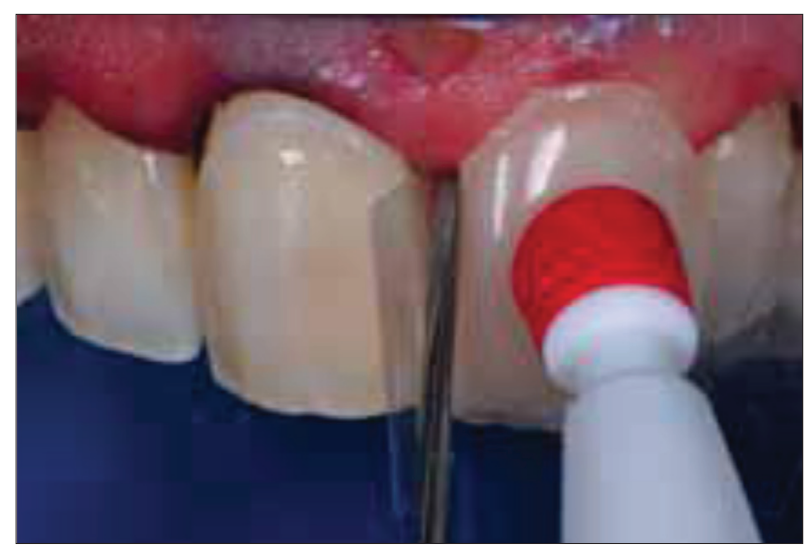

Figure 8. Silicone instrument used for adaptation of composite veneer

Slika 8. Silikonski instrument koji se koristi za adaptaciju kompozitne fasete

the palatal and labial side. Final polishing was done using flexible aluminium disks.

\section{Case 2}

The patient aged 23 visited the Clinic for Restorative Dentistry and Endodontics dissatisfied with the appearance of one of her teeth in the anterior region. During the clinical check up of the tooth 12, a deviation of the lateral incisor was found (Figure 9). The decision was to reconstruct her tooth with the Componeer system. After cleaning the tooth surface, the shade of dentin and enamel was determined using the shade key under the daylight. Size M of ComponeerTM Contour Guide that is unique and transparent precise contour for the proper form of teeth was chosen. One Coat Bond was applied on the inner surface of the composite veneer and left without light polymerization. The tooth was etched with 35\% phosphoric acid for 30 seconds and rinsed, and then bond applied, dried and polymerized for 20 seconds. Following was the application of the composite color BRILLIANT EverGlow A1/B1 (Coltene/Whaledent) and the color Componeer was applied with a specialized spatula on the inner surface of the veneer without any pressure. The composite veneer was hold with a specially designed holder and then applied on the tooth using specialized silicone instrument (Figure 10). Polymerization was applied 40 seconds on both palatal and labial surface. Polishing and finishing was done with flexible aluminium disks. High esthetic results were achieved with the use of composite BRILLIAND EverGlow (Coltene/Whaledent) that correspond to the composite veneer color (Figure 11).

\section{DISCUSSION}

Contemporary esthetic dentistry can treat simple and complex cases with various alternatives. Prior to doing esthetic restoration, indication and proper diagnosis must be carefully determined [13]. Every case is different and treatment planning must be based on individual needs. In

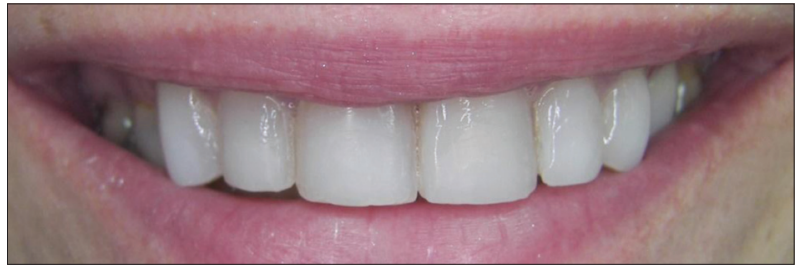

Figure 9. Final look of the teeth after restoration with the Componeer system

Slika 9. Finalni izgled zuba po završetku restorativnog postupka

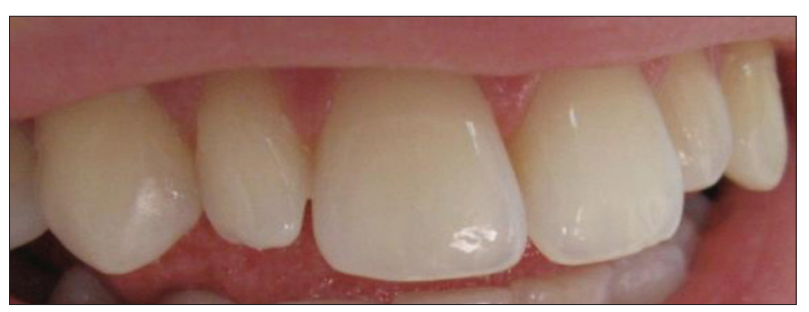

Figure 10. Deviated aesthetics and morphological deviation of lateral incisive

Slika 10. Narušena estetika i morfološka nepravilnost lateralnog inciziva

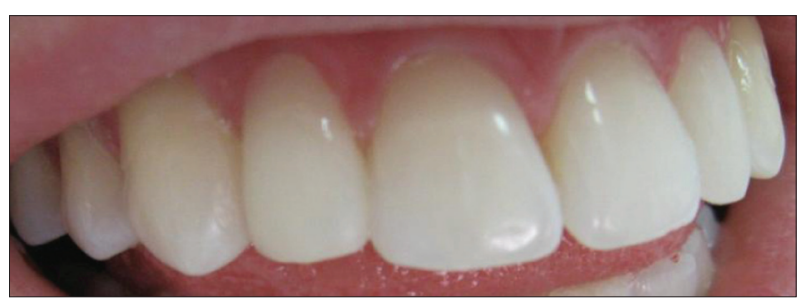

Figure 11. Final restoration with Componeer

Slika 11. Definitivna restauracija kompozitnom fasetom

both presented cases, new composite system was chosen over direct restoration due to shorter chair time and easier technique. Compared to direct restorations, prefabricated composite veneers have advantages, such as reduced polymerization stress, lower gap formation, post-operation hypersensitivity, micro-leakage and optimum marginal adaptation [14]. Strong adhesion of two composites was optimally obtained with the micro-retentive surface of the composite veneer $(2 \mathrm{~nm})$. The Componeer veneer (Coltene/Whaledent) premade of nano-hybrid composite not only makes esthetic restoration faster but also offers new optimal, functional and economical esthetics. Enamel composite veneers are available in various sizes, from small, medium, large to extra-large, and two transparent colors: neutral (universal) and light (white transparent). With the appropriate dentin color that is under the enamel composite veneer, various combinations of colors can be achieved. The composite veneer is made of composite material so additional esthetics can be achieved with regular composite material. Also, if veneer is damaged, it can be easily restored with composite material as well.

Clinical technique described in this article shows that it can be done routinely on anterior teeth, for correction of malpositioned teeth, diastema and discoloration [15, 16]. Prefabricated composite veneers present acceptable esthetic solution that is more acceptable than traditional porcelain veneers. 


\section{CONCLUSION}

New composite system Componeer is easy to use and restoration can be done during only one visit. Excellent esthetic results with optimal adjustment of colors, forms and structures can be achieved without the need for laboratory work. Componeer composite veneers provide new options of functionality, economy and esthetics.

\section{REFERENCES}

1. Baratieri LN, Araujo EM Jr, Monteiro S Jr. Composite Restorations in Anterior Teeth: Fundamentals and Possibilities. Chicago: Quintessence; 2005.

2. Culp L. Replicating natural dentition with composite resin. Pract Proced Aesthet Dent. 2004; 16:27-30.

3. de Araujo Junior EM, Baratieri LN, Monteiro Junior S, Vieira LC, de Andrada MA. Direct adhesive restoration of anterior teeth. Part 1. Fundamentals of excellence. Pract Proced Aesthet Dent. 2003; 15(3):233-40. [PMID: 12956050]

4. de Araujo Junior EM, Baratieri LN, Monteiro Junior S, Vieira LC, de Andrada MA. Direct adhesive restoration of anterior teeth: Part 2. Clinical protocol. Pract Proced Aesthet Dent 2003; 15(5):351-7. [PMID: 12926402]

5. Ferracane JL. Resin composite - state of the art. Dent Mater. 2011; 27(1):29-38. [DOI: 10.1016/j.dental.2010.10.020] [PMID: 21093034]

6. Dietschi D. Free-hand bonding in the esthetic treatment of anterior teeth: Creating the illusion. J Esthet Dent. 1997; 9(4):156-64. [DOI: 10.1111/j.1708-8240.1997.tb00936.x] [PMID: 9468879]

7. Dietschi D. Free-hand composite resin restorations: A key to anterior aesthetics. Pract Periodontics Aesthet Dent. 1995; 7(7):15-25. [PMID: 9002903]
8. Dietschi D. Layering concepts in anterior composite restorations. J Adhes Dent. 2001; 3(1):71-80. [PMID: 11317387]

9. Sowmya K, Dwijendra KS, Pranitha V, Roy KK. Esthetic Rehabilitation with Direct Composite Veneering: A Report of 2 Cases. Case Rep in Dent. 2017; 2017:7638153. [DOI: 10.1155/2017/7638153] [PMID: 28484652]

10. Sensi LG, Marson FC, Roesner, TH, Baratieri LN, Monteiro S Jr. Fluorescence of composite resins: Clinical considerations. Quintessence Dent Technol. 2006; 29:43-53.

11. Dietschi D, Devigus A. Prefabricated composite veneers: Historical perspectives, indications and clinical application. Eur J Esthet Dent. 2011; 6(2):178-87. [PMID: 21734966]

12. Gomes G, Perdigao J. Prefabricated composite resin veneers - A clinical review. J Esthet Restor Dent. 2014; 26(5):302-13. [DOI: 10.1111/jerd. 12114] [PMID: 24942614]

13. Ahmed KE, Murbay S. Survival rates of anterior composites in managing tooth wear: systematic review. J Oral Rehabil. 2015; 43(2):145-53. [DOI: 10.1111/joor.12360] [PMID: 26440584]

14. El Zohairy AA, De Gee AJ, Mohsen MM, Feilzer AJ. Microtensile bond strength testing of luting cements to prefabricated CAD/CAM ceramic and composite blocks. Dent Mater. 2003; 19(7):575-83. [DOI: 10.1016/S0109-5641(02)00107-0] [PMID: 12901980]

15. Perdigao J, Sezinando A, Munoz MA, Luque-Martinez IV, Loguercio AD. Prefabricated veneers - Bond strengths and ultramorphological analyses. J Adhes Dent. 2014; 16(2):137-46. [DOI: 10.3290/j. jad.a30689] [PMID: 24179983]

16. Dietschi D. Optimizing smile composition and esthetics with resin composites and other conservative esthetic procedures. Eur J Esth Dent. 2008; 3(1):14-29. [PMID: 19655556] 


\title{
Minimalno restaurativni tretman direktnim kompozitnim fasetama - prikaz slučaja
}

\author{
Sonja Apostolska', Vasilka Rendžova', Marina Eftimoska', Vesna Filipovska', Sašo Elenčevski², \\ Nadica Janeva² \\ 'Univerzitet „Sv. Kiril i Metodij”, Stomatološki fakultet, Klinika za bolesti zuba i endodonciju, Skoplje, Republika Makedonija; \\ 2Univerzitet „Sv. Kiril i Metodij”, Stomatološki fakultet, Klinika za mobilnu protetiku, Skoplje, Republika Makedonija
}

\begin{abstract}
KRATAK SADRŽAJ
Estetska i minimalno invazivna tehnika su danas dve vrlo značajne teme restaurativne stomatologije. Estetski zahtevi sa savremenim kompozitnim materijalima i moderna adhezivna tehnologija omogućavaju dizajniranje estetskih restauracija bez invazivnih preparacija zuba, koje su uobičajene za protetske nadoknade. Savremena estetska stomatologija danas rešava i jednostavne i kompleksne slučajeve različitim tehikama restauracije.

Ključne reči: kompozitne fasete; adhezivna tehnika; estetika
\end{abstract}

\section{UVOD}

Estetska i minimalno invazivna tehnika su danas dve glavne teme restaurativne stomatologije. Restaurativna tehnika, koja koristi savremene kompozitne materijale i modernu adhezivnu tehnologiju, pruža brojne opcije za dizajniranje estetske restauracije bez invazivnih preparacija zuba $[1,2]$. Potreba za estetskim tretmanom proizilazi kako iz različitih sistemskih preduslova, tako i iz različitih genetskih, dentalnih i traumatoloških razloga $[3,4,5]$. Ključni faktori za rešavanje ovih slučajeva su kompozitni materijali i adhezivni sistemi. Slojevita tehnika, primenom različitih nijansi, omogućava odlične biomimetičke restauracije. Zbog toga je neophodno poznavati karakteristike materijala, kako bi se ostvario maksimalan estetski i funkcionalni efekat [6]. Moderni kompoziti omogućavaju rekonstrukciju spoljašnjeg oblika i boje, kao i reprodukciju unutrašnje strukture zuba. Estetska restauracija danas može imitirati optička svojstva prirodnog zuba $[7,8]$. Nanotehnologija u proizvodnji kompozitnih materijala omogućava veliki izbor boja i dosta izražen „,kameleonski efekat". Omogućava dobro modelovanje ispuna i izradu složenih restauracija zuba i u frontalnoj i u posteriornoj regiji.

Klinički postupak izrade direktnih kompozitnih restauracija uključuje: poštovanje individualnih morfoloških karakteristika zuba, izbor odgovarajuće nijanse materijala, pravilnu i minimalnu preparaciju zuba, slojevitu tehniku aplikacije i završno finiranje i poliranje. Primenom slojevite tehnike dobija se odlična anatomska forma zuba, ali je problem što je za to potrebno mnogo vremena. Isto tako, kao problem se nameće i činjenica da se tokom vremena pojavljuju tamna polja, zbog vazdušnih mehurića pri aplikaciji kompozitnog materijala, što kasnije dovodi do promene boje cele restauracije $[9,10]$.

Svi ovi problemi su danas prevaziđeni novim kompozitnim sistemom, koji kombinuje prednosti direktnih kompozitnih restauracija. To je novi sistem koji omogućava restauraciju anteriornih zuba pomoću gotovih kompozitnih faseta. Ovaj sistem omogućava da se zubi ne samo restauriraju već i da se promeni njihov oblik. Primenom ovog sistema obezbeđuju se izvanredne mogućnosti za restauraciju frakturiranih i destruiranih zuba, morfoloških i strukturnih nepravilnosti, diskoloriranih zuba i dijastema u frontalnoj regiji, odnosno karijesa, trauma, erozija, abrazija, fraktura i manjih ortodontskih nepravilnosti $[11,12]$. Direktni kompozitni sistem nudi novu i zanimljivu dimenziju tretmana, koji je za pacijenta i za stomatologa ekonomičniji,jer se izvodi u tokom jedne posete.

Cilj ovog rada je bio da se na osnovu prikaza slučaja objasni anteriorna rekonstrukcija zuba primenom sistema Componeer.

\section{PRIKAZ SLUČAJA 1}

Pacijentkinja starosti 40 godina došla je na Kliniku za bolesti zuba i endodonciju jer nije bila zadovoljna izgledom svojih zuba $\mathrm{u}$ frontalnoj regiji. Kliničkim pregledom zuba 13, 12, 11, 21, 22, 23 uočene su neadekvatna morfologija i neadekvatne restauracije (Slika 1). Odlučili smo da zube estetski rekonstruišemo primenom direktnih kompozitnih faseta sistemom Componeer. Nakon čišćenja površine zuba određena je gleđna i dentinska boja pomoću ključa pod dnevnim svetlom. Pomoću ComponeerTM Contour Guide izabrani su veličina i oblik zuba (izabrali smo veličinu L) (Slika 2 i 3). Posle selekcije veličine i oblika uzeli smo odgovarajuću gleđnu fasetu, koju smo minimalno korigovali diskom (Swiss Flex, Coltene), sa malim brojem obrtaja, bez upotrebe vode, zbog marginalne adaptacije (Slika 4). Zatim je urađena minimalna preparacija (jer je debljina fasete svega 0,3 $\mathrm{mm}$ ) i odstranjena stara restauracija (Slika 5 i 6). One Coat Bond smo naneli na unutrašnju površinu Componeera i ostavili bez svetlosne polimerizacije. Zube smo nagrizali sa 35-procentnim rastvorom fosforne kiseline 30 sekundi i isprali, zatim naneli One Coat Bond na površinu zuba, osušili i polimerizirali 20 sekundi. Nakon toga naneli smo dentinsku kompozitnu boju BRILIANT EverGlow A2/B2 na površinu zuba, a gleđna boja je aplikovana na unutrašnju površinu Componeera bez prekomernog pritiska, pomoću specijalne špatule. Kompozitna faseta je držana specijalno dizajniranim držačem, a zatim smo je adaptirali na zub pritiskom sa specijalnim silikonskim instrumentom (Slika 7 i 8). Posle provere okluzije i centralne linije inciziva urađena je polimerizacija prvo sa palatinalne strane (u cilju smanjenja stresa od polimerizacije), a zatim labijalno. Polimerizacija je trajala 40 sekundi i sa palatinalne i sa labijalne strane. Definitivna obrada je urađena fleksibilnim aluminijumskim diskovima.

\section{PRIKAZ SLUČAJA 2}

Pacijentkinja starosti 23 godine takođe je bila nezadovoljna izgledom svojih zuba u frontalnoj regiji. Na kliničkom pregledu 
zuba uočena je morfološka nepravilnost lateralnog inciziva (Slika 2). Odlučili smo da zub estetski rekonstruišemo primenom sistema Componeer. Nakon čišćenja površine zuba određena je gleđna i dentinska boja pomoću ključa pod dnevnim svetlom. Pomoću ComponeerTM Contour Guide izabrali smo srednju veličinu. Posle selekcije veličine i oblika One Coat Bond je nanet na unutrašnju površinu kompozitnog laminata i ostavljen bez svetlosne polimerizacije. Zub smo nagrizali sa 35-procentnim rastvorom fosforne kiseline tokom 30 sekundi i isprali, zatim naneli bond na površinu zuba, osušili i polimerizirali 20 sekundi. Nakon toga dentinsku kompozitnu boju A1/B1 BRILIANT EverGlow (Coltene/Whaledent) naneli smo na površinu zuba, a gleđna boja je naneta na unutrašnju površinu Componeera, bez prekomernog pritiska, specijalnom špatulom. Kompozitna faseta je držana specijalno dizajniranim držačem (Slika 3), a zatim je adaptirana na zub pritiskom specijalnim silikonskim instrumentom. Nakon provere aplikacije fasete urađena je polimerizacija, u trajanju od 40 sekundi, i sa palatinalne i sa labijalne strane. Definitivna obrada je urađena sa fleksibilnim aluminijumskim diskovima. U cilju postizanja odgovarajuće estetike preporučuje se upotreba kompozita BRILIANT EverGlow (Coltene/Whaledent), jer odgovara boji kompozitne fasete (Slika 2 a).

\section{DISKUSIJA}

Savremena estetska stomatologija danas rešava i jednostavne i kompleksne slučajeve različitim alternativama. Pre izrade estetske restauracije potrebno je definisati indikaciju i odrediti dijagnostički cilj [13]. Ne treba se uvek odlučiti za najbolje rešenje, već treba izabrati perfektnu soluciju za svaki pojedinačan slučaj. Direktne kompozitne restauracije bi mogle biti dobar izbor za oba slučaja, međutim, mi smo se odlučili za novi kompozitni sistem zbog skraćenog vremena rada i jednostavnije tehnike izrade. Kada uporedimo direktne restauracije, prefabrikovane kompozitne fasete imaju prednosti jer se redukuje polimerizacijski stres, smanjuje formiranje mikropukotine na spoju zuba i restauracije i eliminiše postoperativna hipersenzitivnost i mikropropuštanje [14]. Dobra adhezibna veza dva kompozita postiže se primenom mikroretentivne površine kompozitne fasete (2 nm). Kompozitni sistem Componeer (Coltene/Whaledent) izrađen je od nanohibridnog kompozita i estetsku restauraciju čini lakšom i efikasnijom, ali i funkcionalnom i ekonomičnom. Gleđne kompozitne fasete su dostupne u različitim veličinama (od malih, srednjih, velikih do ekstravelikih) i u dve različite transparentne boje ((neutralna (univerzalna) i svetla (transparentno bela)). Odgovarajućom dentinskom bojom, koja se nalazi ispod gleđnih kompozitnih faseta, mogu se kreirati različite kombinacije boja. Zbog toga što je kompozitna faseta izrađena od kompozitnog materijala ovde se mogu kreirati mamelomi na površini zuba i ako nastanu oštećenja, mogu se jednostavno restaurirati kompozitom. Klinička tehnika koja je opisana u ovim slučajevima ukazuje da se korekcija može raditi rutinski kod anteriornih zuba (korekcija malpozicioniranih zuba, dijastema i korekcija diskoloracija) $[15,16]$. U ovoj kliničkoj studiji akcent je dat na primeni onih kompozitnih faseta koje daju zadovoljavajući estetski izgled i koje su prihvatljivije od tradicionalnih porcelanskih faseta.

\section{ZAKLJUČAK}

Naše kliničko iskustvo daje nam pravo da zaključimo da primena kompozitnih faseta omogućava laku i efikasnu primenu, i uz samo jednu posetu obezbeđuje kvalitetne dentalne restauracije sa odličnim estetskim rezultatima. Primena kompozitnih faseta obezbeđuje optimalnu i jednostavnu restauraciju i pruža nove opcije za funkcionalnu, ekonomičnu estetiku, koja je najveća olakšica za pacijente. 